\title{
Destruction of Anderson localization by a weak nonlinearity
}

\author{
A.S.Pikovsky ${ }^{1}$ and D.L.Shepelyansky ${ }^{2,1}$ \\ ${ }^{1}$ Department of Physics, University of Potsdam, Am Neuen Palais 10, D-14469, Potsdam, Germany \\ ${ }^{2}$ Laboratoire de Physique Théorique, UMR 5152 du CNRS, Université Toulouse III, 31062 Toulouse, France
}

(Dated: November 1, 2018)

\begin{abstract}
We study numerically a spreading of an initially localized wave packet in a one-dimensional discrete nonlinear Schrödinger lattice with disorder. We demonstrate that above a certain critical strength of nonlinearity the Anderson localization is destroyed and an unlimited subdiffusive spreading of the field along the lattice occurs. The second moment grows with time $\propto t^{\alpha}$, with the exponent $\alpha$ being in the range $0.3-0.4$. For small nonlinearities the distribution remains localized in a way similar to the linear case.
\end{abstract}

PACS numbers: 05.45.-a, 03.75.Kk, 05.60.Gg, 05.30.Jp

The Anderson localization [1] has been originally discussed for electron propagation in a disordered potential. Nowadays, an impressive technological progress in experiments with cold atoms allows one to create a disordered quasi-1D potential by laser fields and to detect signatures of localization of a Bose-Einstein condensate (BEC) in presence of disorder [2, 3, 4, 5, 6]. An interesting new aspect in such systems is an importance of nonlinear effects since in a good approximation the evolution of BEC can be described by the nonlinear Gross-Pitaevskii equation (see e.g. [7]). An interplay of disorder, localization, and nonlinearity appears also in other physical systems like wave propagation in nonlinear disordered media (see e.g. [8, 9]), chains of nonlinear oscillators (see e.g. [10]) with randomly distributed frequencies, and models of quantum chaos with a kicked soliton [11] and a kicked BEC 12, 13, 14.

We focus here on the discrete Anderson nonlinear Schrödinger equation (DANSE)

$$
i \hbar \frac{\partial \psi_{n}}{\partial t}=E_{n} \psi_{n}+\beta\left|\psi_{n}\right|^{2} \psi_{n}+V\left(\psi_{n+1}+\psi_{n-1}\right)
$$

where $\beta$ characterizes nonlinearity, $V$ is hopping matrix element, on-site energies are randomly distributed in the range $-W / 2<E_{n}<W / 2$, and the total probability is normalized to unity $\sum_{n}\left|\psi_{n}\right|^{2}=1$. For $\beta=0$ and weak disorder all eigenstates are exponentially localized with the localization length $l \approx 96(V / W)^{2}$ at the center of the energy band [19]. Hereafter we set for convenience $\hbar=V=1$, thus the energy coincides with the frequency.

For nonlinear equation (1) we consider the following problem: how an initially localized field $\left|\psi_{n}(0)\right|^{2}=\delta_{n, 0}$ is spreading? In the linear case the spreading saturates after excitation of all linear modes that have significant values at $n=0$. The same process of "initial excitation" of modes happens in the nonlinear case as well, this initial stage of spreading has been analyzed recently in refs. [20, 21] and is now well understood. However, a behavior at large time scales remains less clear. The results presented in 20] support the view of eventual exponential localization of the field. We demonstrate below that the spreading is unlimited, however it is rather slow - subdiffusive.

The basic idea is to use the equivalence between the Anderson localization and the localization of quasienergy eigenstates in a kicked quantum rotator [15, 16]. In the latter model the case of quantum chaos with nonlinearity has been considered analytically and numerically in [17, 18] and it has been shown that above a certain nonlinearity level, nonlinear phase shifts lead to a complete delocalization with a subdiffusive spreading over all states [17]. Furthermore it has been argued that the same situation should appear for the DANSE (1).

We first apply the theoretical arguments of paper [17] to model (1), and then perform large scale numerical simulations of a wave packet spreading on a time scale which is by 5-6 orders of magnitude larger compared to that in [20]. Our results are in general consistent with the theory developed for the quantum chaos model [17] that predicts unlimited subdiffusive spreading.

At first glance, the effect of nonlinearity seems to be vanishing in the limit of a broadly spread distribution. Indeed, if the field is spread over $\Delta n$ sites, then due to the conservation of the total probability in Eq. (10) the field is small $\left|\psi_{n}\right|^{2} \approx 1 / \Delta n$ and correspondingly small are the nonlinear effects. However, one should compare the nonlinear frequency shift $\delta \omega \sim \beta\left|\psi_{n}\right|^{2}$ with the characteristic distance $\Delta \omega$ between frequencies of excited modes (the latter are the exponentially localized modes of the linear disordered lattice). As the number of these modes is proportional to $\Delta n$, the distance between the frequencies obeys $\Delta \omega \sim 1 / \Delta n$, and the relative nonlinear frequency shift $\delta \omega / \Delta \omega \sim \beta$ is independent of the width of the field distribution but is proportional to the nonlinearity parameter $\beta$. This means that the effect of nonlinearity does not qualitatively depend on the width of field distribution: if for some $\beta>\beta_{c}$ the field is chaotic, chaos remains while spreading, and no transition to regularity that blocks spreading is expected; for small nonlinearities $\beta<\beta_{c}$ the dynamics which is nearly regular (KAM regime) for localized distributions remains as such for all times. However, quantitative difference 
can occur and the spreading can slow down for wide distributions. Again, to roughly estimate this effect, we can adopt here the arguments of 17]. In the basis of linear localized modes, the evolution of the amplitudes $C_{m}$ of these modes is due to their nonlinear coupling, i.e. $\dot{C}_{m} \sim \beta C_{m_{1}} C_{m_{2}} C_{m_{3}}$. Assuming randomness of the phases, we can estimate the rate of excitation of a newly involved mode as $\sim|C|^{6} \sim 1 /(\Delta n)^{3}$. On the other hand, excitation of a new mode is none other than diffusive spreading of the field, thus $\frac{d}{d t}(\Delta n)^{2} \sim 1 /(\Delta n)^{3}$. Solution of this equation yields subdiffusive spreading

$$
(\Delta n)^{2} \propto t^{2 / 5} .
$$

For numerical simulations we used the operator splitting integration scheme for the time evolution given by (11): $\psi_{n}(t+\Delta t)=\hat{R} \hat{S} \psi_{n}(t)$, where $\hat{R}=\exp \left(-i\left(E_{n}+\right.\right.$ $\left.\left.\beta\left|\psi_{n}\right|^{2}\right) \Delta t\right)$ is local and $\hat{S}=\exp (-2 \Delta t \cos \hat{\theta})$ is nontrivial because $\hat{\theta}$ is the operator conjugated to $\hat{n}=-i \partial / \partial \theta$. This kick-like integration scheme is unitary and preserves the total probability. In addition it introduces high harmonics with frequencies $\omega_{m}=m 2 \pi / \Delta t$ and integer $m$. However, at small $\Delta t$ these frequencies are significantly larger than the system energy band width $B$ : $\omega_{1}=2 \pi / \Delta t \gg B=(4+W)$ and their average effect should be exponentially small. We have chosen $\Delta t=0.1$ that gives high frequency oscillations of total energy on a percent level. A further decrease of $\Delta t$ by an order of magnitude does not affect the average behavior of the field spreading.

We used two discrete implementations for the evolution operator $\hat{S}$ of the linear Schrödinger equation. In the first one we represent $\hat{S}$ as a band matrix whose elements are Bessel functions. At $\Delta t=0.1$ we keep Bessel functions $J_{m}(2 \Delta t)$ with $|m| \leq 10$ that preserves probability on one integration step with the accuracy better than $10^{-16}$, whereas after time $t=10^{8}$ the probability is preserved with accuracy better than $10^{-7}$. For one disorder realization such a run with the total number of sites $N=2001$ and $t=10^{8}$ takes about 6000 mins of CPU on a $4 G H z$-workstation. In another implementation we used the unitary Crank-Nicholson scheme 22]. The results obtained by both methods are very similar; below mainly those from the first method are presented because of its better efficiency.

To characterize the wave packet spreading over the lattice sites we compute its average squared width, i.e. the second moment $\left\langle(\Delta n)^{2}\right\rangle=\sigma(t)=\sum_{n}(n-\langle n\rangle)^{2}\left|\psi_{n}(t)\right|^{2}$. The averaging over disorder realizations was performed for the $\log$ arithm of this quantity, i.e. for $\log \sigma$. The dependence of the averaged $\sigma$ on time $t$ is shown in Fig. 1 for a moderate nonlinearity $\beta=1$ and disorder strengths $W=2$ and $W=4$. It clearly shows a subdiffusive spreading

$$
\sigma(t) \propto t^{\alpha}
$$

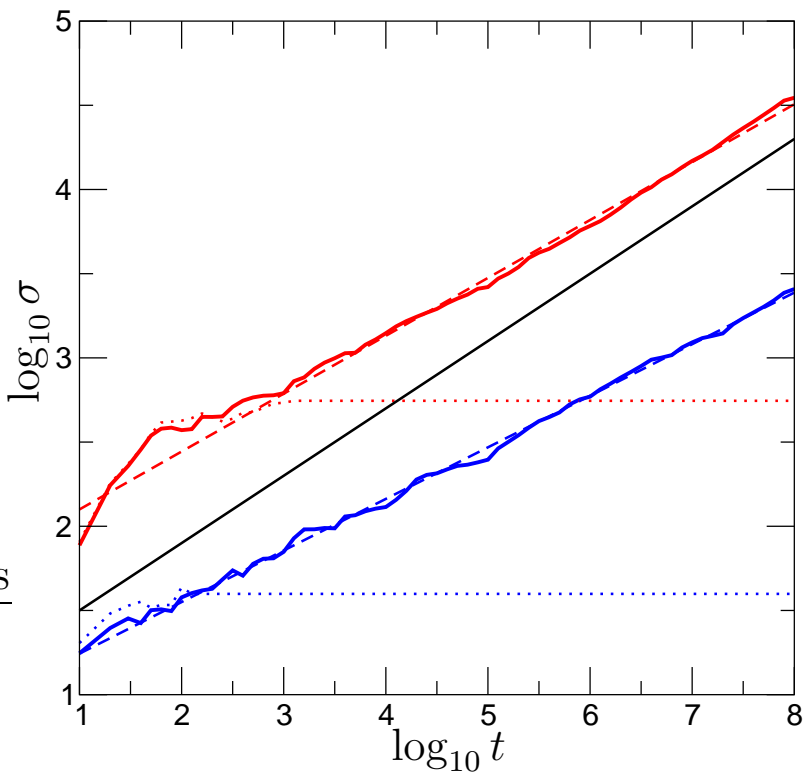

FIG. 1: (color online) The dependence of the second moment $\sigma$ of the probability distribution $w_{n}$ on time $t$ for two disorder strengths $W=2$ (top full red/gray curve) and $W=4$ (bottom full black/blue curve) for $\beta=1$; dotted curves of same color show data at $\beta=0$ (for large $t$ only the average value is shown by a horizontal line). The values of $\log _{10} \sigma$ are averaged over 8 disorder realizations and over time intervals $\Delta\left(\log _{10} t\right) \approx 0.1$. Dashed lines show the numerical fits $\log _{10} \sigma=\alpha \log _{10} t+\eta$ with $\alpha=0.344 \pm 0.003, \eta=1.76 \pm 0.02$ (done for $3 \leq \log _{10} t \leq$ 8 for $W=2$ ) and $\alpha=0.306 \pm 0.002, \eta=0.94 \pm 0.01$ (done for $2 \leq \log _{10} t \leq 8$ for $\left.W=4\right)$. The full straight line shows the slope $\alpha=0.4$. Here and below the logarithms are decimal.

which continues without any sign of saturation up to extremely large time $t_{\max }=10^{8}$. At $t_{\max }$ the variance $\sigma$ becomes by two orders of magnitude larger than its saturation value at $\beta=0$. Yet the initial spreading at $t \lesssim l$ for $\beta=1$ is similar to the linear case $\beta=0$ in agreement with 21]. The exponent $\alpha$ was determined by a fit in the time interval $t_{0}<t<t_{\max }$, where $t_{0}$ is the characteristic time at which the linear spreading ends: $\sigma(\beta=0 ; t) \lesssim \sigma\left(\beta, t_{0}\right)$. The fits are shown in Fig. 1 and the fit values are given in the caption to Fig. 1. The statistical error in the value of $\alpha$ is rather small due to a large time interval, however $\alpha$ values for individual realizations fluctuate rather strongly varying in the interval $0.32 \leq \alpha \leq 0.39$ and $0.28 \leq \alpha \leq 0.41$ with the standard deviation errors $\Delta \alpha / \alpha=0.026$ and 0.045 , for $W=2$ and 4 respectively. There are also certain time variations, e.g. for $10^{5} \leq t \leq 10^{8}$ the fits give $\alpha=0.375$ and 0.319 for $W=2$ and 4 respectively. In spite of these variations the value of $\alpha$ differs visibly from the theoretically expected value $\alpha=0.4$ (Ref. [17] and Eq. (2)). We will return to the discussion of this deviation later.

A more detailed characterization of the field spreading is given by the probability distribution $w_{n}=\left|\psi_{n}(t)\right|^{2}$. We show $\log _{10} w_{n}$ averaged over disorder realizations in 


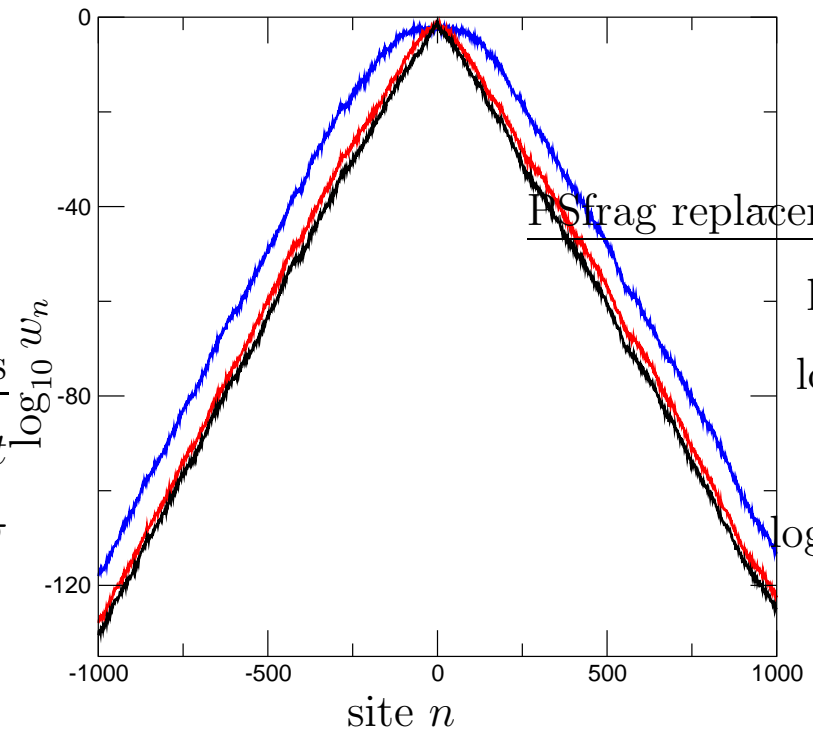

FIG. 2: (color online) Probability distribution $w_{n}$ over lattice sites $n$ at $W=4$ for $\beta=1, t=10^{8}$ (top blue/solid curve) and $t=10^{5}$ (middle red/gray curve); $\beta=0, t=10^{5}$ (bottom black curve; the order of the curves is given at $n=500$ ). At $\beta=0$ a fit $\ln w_{n}=-(\gamma|n|+\chi)$ gives $\gamma \approx 0.3, \quad \chi \approx 4$. The values of $\log _{10} w_{n}$ are averaged over the same disorder realizations as in Fig. 1

Figs. 2, 3 at $t=10^{5}$ and $10^{8}$. For $W=4$ the tails of the probability distribution drop down to enormously small values $\sim 10^{-130}$ that can be reached due to our integration scheme which works efficiently up to very small absolute values of probability. The tails of the distribution $w_{n}$ drop exponentially with the same slope as for the linear case $\beta=0$ which is also shown; the decay rate $\gamma \approx 0.30$ is close to the theoretical value $2 / l \approx 0.33$. Another notable feature of $w_{n}$ is a flat distribution, chapeau, centered near the initially populated site $n=0$. Inside the chapeau the sites are populated in an approximately homogeneous way, and hence its width is essentially determined by the second moment $\sigma(t)$. For $W=2$ the decay rate $\gamma$ for $\beta=0$ drops approximately by a factor $\approx 4$ compared to the case $W=4$, in agreement with the theoretical expression for $l$. Due to a larger value of $l$, the spreading over the lattice sites is broader and a non-exponential shape of the distribution $w_{n}$ at $t=10^{8}$ is more visible. At shorter times $t=10^{5}$ the tails of the distribution are very similar to those in the linear case $\beta=0$.

The dependence of $\sigma(t)$ on nonlinearity $\beta$ is shown in Fig. 4 for $W=4$. One can clearly see that for $\beta=0.03$ the growth of $\sigma$ is stopped completely, the probability distribution $w_{n}$ is close to exponential localization like for $\beta=0$ (see Fig.5). For $\beta=0.1$ there is still a very slow increase of $\sigma$ with time, which is however hardly distinguishable from a saturation. This value of $\beta$ is presumably close to a critical one, at which the unlimited

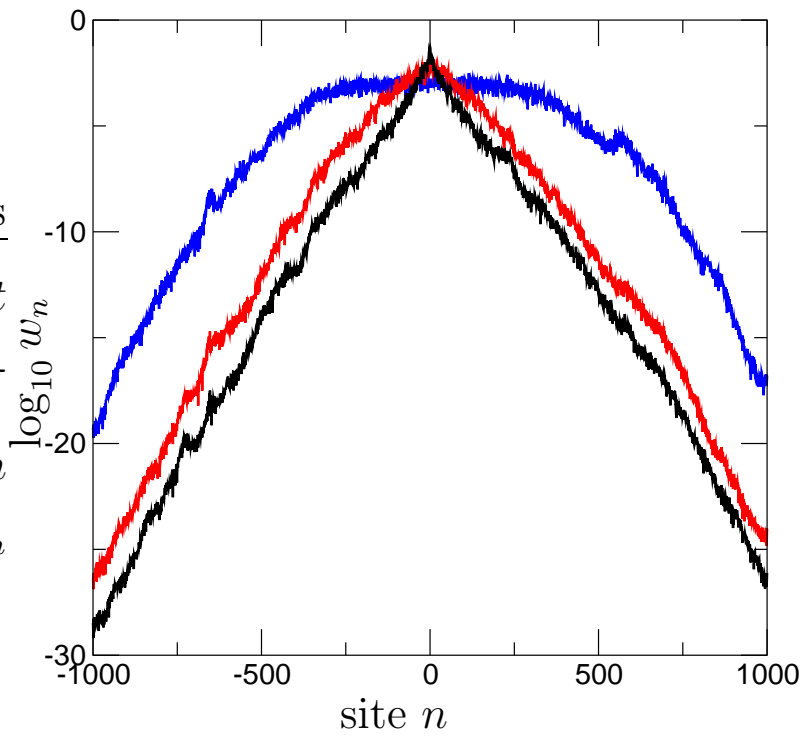

FIG. 3: (color online) Same as in Fig. 2 but with $W=2$. At $\beta=0$ a fit $\ln w_{n}=-(\gamma|n|+\chi)$ gives $\gamma \approx 0.06, \quad \chi \approx$ -3 . The values of $\ln w_{n}$ are averaged over the same disorder realizations as in Fig. 1 .

spreading sets on. A similar transition occurs for $W=2$. These data show that a delocalization transition takes place at a certain critical nonlinearity $\beta_{c} \approx 0.1$. We note that the qualitative and quantitative features of the dynamics seem to be independent of the sign of $\beta$ : the spreading for $\beta=-1$ is similar to that for $\beta=1$ (we have checked this also for $\beta= \pm 2$ ).

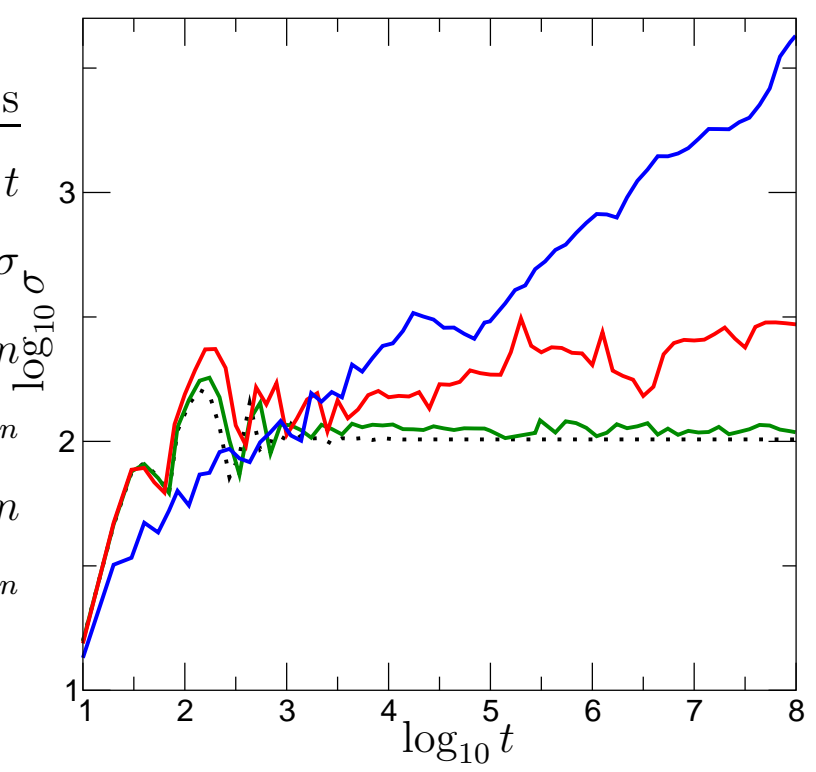

FIG. 4: (color online) Dependence of the second moment $\sigma$ of probability distribution $w_{n}$ on time $t$ for different strengths of nonlinearity $\beta=1,0.1,0.03,0$ at $W=4$ (curves from top to bottom at $\log _{10} t=4.5$, respectively). Data are shown for one particular disorder realization. 


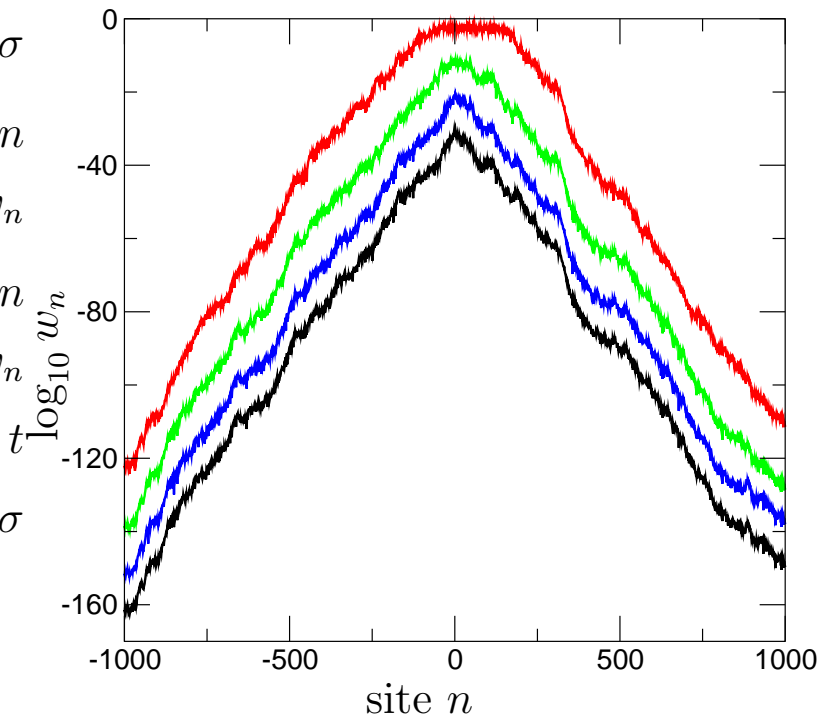

FIG. 5: (color online) Probability distribution $w_{n}$ over lattice sites $n$ for the same disorder realization as in Fig. 4. for $W=$ $4, t=10^{8}$ and for different nonlinearities $\beta=1,0.1,0.03,0$ (from top to bottom; for clarity 3 bottom curves have additional vertical shifts $-10,-20$ and -30 compared to the top one).

The obtained results show close similarities between the DANSE model and the nonlinear kicked rotator studied in [17]. In both cases for $\beta>\beta_{c}$ a subdiffusive spreading over the lattice continues up to enormously long times. In both models the probability distribution has a chapeau with approximately homogeneous probability distribution. Outside of the chapeau the probability drops exponentially. The width of the chapeau grows in a subdiffusive way and the exponent $\alpha$ of this growth is approximately the same for both models. The question about the exact value of the exponent remains open. It is possible that at $W=4$ the localization length is relatively short and there are deviations from the theoretical value $\alpha=0.4$ given in [17]. Longer computations with a better statistical averaging are needed to determine the exact value of $\alpha$; the latter may also depend on the parameters $\beta$ and $W$. In particular, slower diffusion might be due to inhomogeneities of the effective mode-to-mode hopping rates, which are more pronounced for smaller localization lengths, i.e. for larger disorder $W$. At the same time the obtained numerical data clearly show the existence of unlimited spreading over the lattice for $\beta>\beta_{c}$. Indeed, for the data of Fig. 1 at $W=2$ the nonlinear frequency shift $\delta \omega \approx \beta w_{n} \approx \beta / \sqrt{\sigma\left(t_{\max }\right)} \approx 0.006$ is much smaller than the typical level spacing between localized states $\delta \nu \approx B / l \approx 0.25$. The same is true for $W=4$. This means that in simulations we have reached the time scales with apparently asymptotic behavior.

In conclusion, we have demonstrated that in a onedimensional nonlinear disordered lattice the Anderson localization is destroyed and the field spreads subdiffusively far beyond the linear localization range. This effect appears to have a threshold in the nonlinearity coefficient, although the transition might be not perfect, as even for small nonlinearities an extremely slow spreading of the field due to Arnold diffusion mechanism is not excluded. It appears also promising to look for other manifestations of nonlinearity-induced destruction of localization, e.g. in the scattering problem [23].

We thank S.Aubry and S. Flach for useful discussions.

[1] P.W. Anderson, Phys. Rev. 109, 1492 (1958).

[2] J.E. Lye, L. Fallani, M. Modugno, D.S. Wiersma, C. Fort, and M. Inguscio, Phys. Rev. Lett. 95, 070401 (2005).

[3] D. Clément, A.F. Varón, M. Hugbart, J.A. Retter, P. Bouyer, L. Sanchez-Palencia, D.M.Gangardt, G.V.Shlyapnikov, and A. Aspect, Phys. Rev. Lett. 95, 170409 (2005).

[4] C. Fort, L. Fallani, V. Guarrera, J.E. Lye, M. Modugno, D.S. Wiersma, and M. Inguscio, Phys. Rev. Lett. 95, 170410 (2005).

[5] T.Sculte, S. Drenkelforth, J.Kruse, W.Ertmer, J. Arlt, K. Sacha, J.Zakrzewski, and M. Lewenstein, Phys. Rev. Lett. 95, 170411 (2005).

[6] D. Clément, A.F. Varón, J.A. Retter, L. SanchezPalencia, A. Aspect, and P. Bouyer, New J. Phys. 8, 165 (2006).

[7] F. Dalfovo, S. Giorgini, L.P. Pitaevskii, and S. Strigani, Rev. Mod. Phys. 71, 463 (1999).

[8] S.E. Skipetrov, and R. Maynard, Phys. Rev. Lett. 85, 736 (2000).

[9] T. Schwartz, G. Bartal, S. Fishman, and M. Segev, Nature 446, 52 (2007).

[10] G. Iooss, and G. James, Chaos 15, 015113 (2005).

[11] F. Benvenuto, G. Casati, A.S.Pikovsky, and D.L.Shepelyansky, Phys. Rev. A 44, R3423 (1991).

[12] C. Ryu, M.F. Andesen, A. Vaziri, M.B. d'Arcy, J.M. Grossman, K. Helmerson, and W.D. Phillips, Phys. Rev. Lett. 96, 160403 (2006).

[13] G. Behinaein, V. Ramareddy, P. Ahmadi, and G.S. Summy, Phys. Rev. Lett. 97, 244101 (2006).

[14] M. Sadgrove, M. Horikoshi, T. Sekimura, and K. Nakagawa, Phys. Rev. Lett. 99, 043002 (2007).

[15] S. Fishman, D. R. Grempel, and R. E. Prange, Phys. Rev. Lett. 49, 509 (1982).

[16] D. L. Shepelyansky, Physica D 28, 103 (1987).

[17] D.L. Shepelyansky, Phys. Rev. Lett. 70, 1787 (1993).

[18] T. Kottos, and M. Weiss, Phys. Rev. Lett. 93, 190604 (2004).

[19] B. Kramer, and A. MacKinnon, Rep. Prog. Phys. 56, 1469 (1993).

[20] L. Sanchez-Palencia, D. Clément, P. Lugan, P. Bouyer, G.V.Shlyapnikov, and A. Aspect, Phys. Rev. Lett. 98, 210401 (2007).

[21] B. Shapiro, Phys. Rev. Lett. 99, 060602 (2007).

[22] W. H. Press et al., Numerical Recipes: the Art of Scientific Computing, Cambridge University Press (1992).

[23] S. Tietsche and A. Pikovsky (in preparation). 